\title{
A New Approach for Texture Classification Based on Average Fuzzy Left Right Texture Unit Approach
}

\author{
Y Venkateswarlu \\ Head Dept. of CSE\&IT \\ Chaitanya Instituteof Engg. \&Tech.,Rajahmundry, India \\ E-mail: yalla_venkat@yahoo.com \\ B Sujatha \\ Assoc. Professor., Dept. of CSE, \\ GIET Rajahmundry, A.P, INDIA \\ E-mail: birudusujatha@gmail.com \\ J V R Murthy \\ Professor, Dept of CSE, JNTU Kakinada \\ E-mail: mjonnalagedda@gmail.com
}

\begin{abstract}
Texture refers to the variation of gray level tones in a local neighbourhood. The "local" texture information for a given pixel and its neighbourhood is characterized by the corresponding texture unit. Based on the concept of texture unit, this paper describes a new statistical approach to texture analysis, based on average of the both fuzzy left and right texture unit matrix. In this method the "local" texture information for a given pixel and its neighbourhood is characterized by the corresponding fuzzy texture unit. The proposed Average Fuzzy Left and Right Texture Unit (AFLRTU) matrices overcome the disadvantage of FTU by reducing the texture unit from 2020 to 79 . The proposed scheme also overcomes the disadvantage of the left and right texture unit matrix (LRTM) by considering the texture unit numbers from all the 4 different LRTM's instead of the minimum one as in the case of LRTM. The cooccurrence features extracted from the AFLRTU matrix provide complete texture information about an image, which is useful for texture classification. Classification performance is compared with the various fuzzy based texture classification methods. The results demonstrate that superior performance is achieved by the proposed method.
\end{abstract}

Index Terms - Texture image, Texton pattern, Classification

\section{INTRODUCTION}

The analysis of texture in images provides an important cue to the recognition of objects. It has been recently observed that different image objects are best characterized by different texture methods. Successful applications of texture analysis methods have been widely found in industrial, biomedical, remote sensing areas and target recognition [1]. Since there are a lot of variations among natural textures, to achieve the best performance for texture analysis or retrieval, different features should be chosen according to the characteristics of texture images. A number of texture analysis methods have been proposed over the years and it is wellrecognized that they capture different texture properties of the image.

To analyze and identify textures, a number of texture analysis methods have been developed. These are autocorrelation function [2], digital transformation [3], textural edges [4], structural elements [2], spatial gray level co-occurrence probability [5], Markov random fields [6], wavelet decomposition methods [7] and so on. The most commonly used approach is the spatial gray level co-occurrence matrix that provides gray-level transition information between two gray levels [8,9]. One of the simplest statistical approaches for describing texture is to use statistical moments of the gray-level histogram of an image or region. However, measures of texture computed using only histograms suffer from the limitation that they carry no information regarding the relative position of pixels with respect to each other.

He and Wang have proposed the texture spectrum (TS) approach for texture analysis $[10,11,12,13]$ that was initially used as a texture filtering approach. The TS methodology [14, 15] has been applied to texture characterization and texture classification showing its promising discrimination performance. But a major inconvenient of this descriptor is the large range of its possible values (there are 6561) at the same time that these values are not correlated. Moreover, as images of the same underlying texture can vary significantly, textural features must be invariant to (large) image 
variations, and at the same time sensitive to intrinsic spatial structures that define textures.

A possible solution to aforementioned problems should be the use of fuzzy logic and fuzzy techniques. Fuzzy logic is a simple and powerful tool to mathematical formalization of ill defined concepts that usually appear at texture analysis and segmentation. On the other hand, fuzzy techniques enable more flexible classifications because elements can have a characteristic only to some degree.

Taur et al. [16] proposed a texture feature based on the fuzzified relative gray levels between pixels. The simplest unit representing a pixel is a 3 -vector called Texture Vector (TV). An image block is represented by the histogram of its TV, and its texture is then characterized using a neural network classifier. Later on, Aina Barcelo et al [17] used the concept of Fuzzy Texture Spectrum (FTS), to be used as the texture feature within texture analysis process. FTS will dramatically reduce the total number of texture units to 2020 . However, the high dimension of 2020 is a computational burden.

The proposed approach in this paper is based on the LRTM [18] and Original TS [10], and tries to alleviate its drawbacks and aforementioned problems. So, to retain its discriminatory power, the proposed method considers a $3 \times 3$ neighbourhood into two connected fuzzy patterns. A connected fuzzy pattern approach gives complete local micro texture information and to avoid the imprecision within the process, we make use of fuzzy logic and fuzzy techniques.

The paper is organized as follows. The concepts of texture unit, and fuzzy texture unit are given in section II. The proposed methodology is given in section III. Section IV contains experimental results and conclusions are given in section $\mathrm{V}$.

\section{TEXTURE UNIT}

In a square raster digital image, each pixel is surrounded by eight neighboring pixels. The local texture information for a pixel can be extracted from a neighborhood of $3 \times 3$ pixels, which represents the smallest complete unit. A general representation of texture elements are shown in Fig.1.

\begin{tabular}{|c|c|c|}
\hline $\mathrm{V}_{1}$ & $\mathrm{~V}_{2}$ & $\mathrm{~V}_{3}$ \\
\hline $\mathrm{V}_{8}$ & $\mathrm{~V}_{0}$ & $\mathrm{~V}_{4}$ \\
\hline $\mathrm{V}_{7}$ & $\mathrm{~V}_{6}$ & $\mathrm{~V}_{5}$ \\
\hline
\end{tabular}

\begin{tabular}{|l|l|l|}
\hline $\mathrm{E}_{1}$ & $\mathrm{E}_{2}$ & $\mathrm{E}_{3}$ \\
\hline $\mathrm{E}_{8}$ & & $\mathrm{E}_{4}$ \\
\hline $\mathrm{E}_{7}$ & $\mathrm{E}_{6}$ & $\mathrm{E}_{5}$ \\
\hline
\end{tabular}

Fig. 1: Representation of texture elements.

A neighborhood of $3 \times 3$ pixels can be denoted by a set containing nine elements: $\mathrm{V}=\left\{\mathrm{V}_{0}, \mathrm{~V}_{1}, \ldots, \mathrm{V}_{8}\right\}$, where $\mathrm{V}_{0}$ represents the intensity value of the central pixel and $\mathrm{V}_{\mathrm{i}}$, where $i=\{1,2, \ldots, 8\}$, is the intensity value of the neighboring pixel i. Based on this the corresponding texture unit (TU) is defined by a set containing eight elements, $\mathrm{TU}=\left\{\mathrm{E}_{1}, \mathrm{E}_{2}, \mathrm{E}_{3}, \mathrm{E}_{4}, \mathrm{E}_{5}, \mathrm{E}_{6}, \mathrm{E}_{7}, \mathrm{E}_{8}\right\}$ where $\mathrm{E}_{\mathrm{i}}$, $\mathrm{i}=1,2,3, \ldots, 8$ is denoted by the following Equation(1).

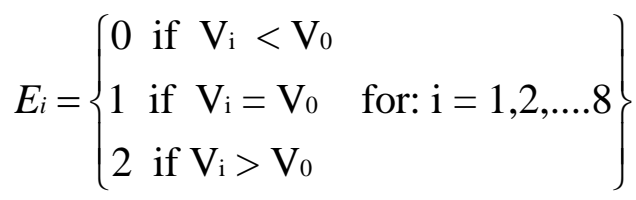

and each element $\mathrm{E}_{\mathrm{i}}$ occupies the same position as the pixel i. As each element of TU has one of the three possible values $(0,1,2)$, the total number of possible TU is $3^{8}=6561$. These texture units are labeled by using the Equation(2)

$\mathrm{N}_{\mathrm{TU}}=\sum_{i=1}^{8} E_{i} 3^{i-1}, \quad \mathrm{~N}_{\mathrm{TU}} \in\left\{0,1,2, \ldots . .\left(\mathrm{N}^{8-1}\right)\right\}$

where $\mathrm{N}_{\mathrm{TU}}$ is the texture unit number. There is no unique way to label and order the 6561 texture units. The high range of TU number makes further process on texture classification is very complicated. To overcome this, the texture units are reduced from 0:6560 to 0:255 for classification purpose by assigning binary value 0 or 1 to each element of TU and by this the corresponding NTU ranges from 0:255 [Ph.D thesis of Dr.V.Vijayakumar].

\section{A. Fuzzy Texture Approach}

The main problem in the above two representations of TU is that they are unable to distinguish between less and far less or greater or far greater and large range of its possible values and moreover these values are not correlated. To address the above and to have high discriminating power, two more membership functions (Greater and Lesser quantities) are introduced in FTS approach. The aim of fuzzy texture unit is to extract local texture information from pixels for representing the texture accurately. To deal accurately with the regions of natural images even in the presence of noise and the different processes of caption and digitization FTS is introduced. For example, even if the human eye perceives two neighboring pixels as equal, they rarely have exactly the same intensity values. However, the desirable situation would be that the TU of homogeneous images contain more number of ones because the human eye can perceive ones. Therefore, if there is lack of ones, the basic TU will take only 0 and 2 values, which means that the real number of possible textures is $2^{8}$, i.e., 256 out of 6561 , and the spectrum will never be totally covered, which misuses the power of TS method.

To overcome the above, the fuzzy membership function is represented as shown in Fig.2 [19]. A texture unit is represented by eight elements each of which has only five possible values $\{0,1,2,3$ and 4$\}$ in fuzzy texture unit approach. The elements are ordered in clockwise around the centre pixel as shown in Fig.1. The following Equation (3) is used to determine the elements, $\mathrm{E}_{\mathrm{i}}$ of the texture unit. In FTU [19], the texture unit is reduced to 2020, so that the computation time is very less when compared to basic approach 


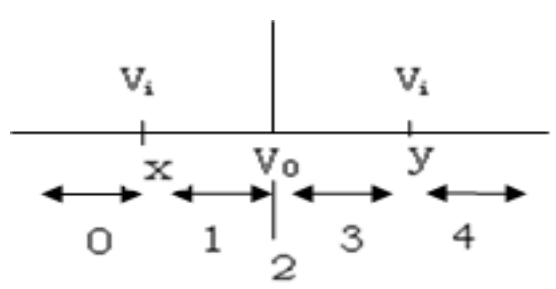

Fig.2: Fuzzy texture number (Base-5) representation.

$E_{\mathrm{i}}=\left\{\begin{array}{lllll}0 & \text { if } & \mathrm{V}_{\mathrm{i}}<\mathrm{V}_{0} & \text { and } & \mathrm{W}_{\mathrm{i}}<x \\ 1 & \text { if } & \mathrm{W}_{\mathrm{i}}<\mathrm{V}_{0} & \text { and } & \mathrm{W}_{\mathrm{i}}>\mathrm{V}_{\mathrm{x}} \\ 2 & \text { if } & \mathrm{W}_{\mathrm{i}}=\mathrm{V}_{0} & & \\ 3 & \text { if } & \mathrm{W}_{\mathrm{i}}>\mathrm{W}_{0} & \text { and } & \mathrm{V}_{\mathrm{i}}>y \\ 4 & \text { if } & \mathrm{V}_{\mathrm{i}}>\mathrm{V}_{0} & \text { and } & \mathrm{V}_{\mathrm{i}}<y\end{array}\right\} \quad$ for $\mathrm{i}=1,2,3, \ldots, 8$

where $\mathrm{x}, \mathrm{y}$ are the user-specified values.

The FTU number $\left(\mathrm{FTU}_{\mathrm{n}}\right)$ is computed as given in Equation (4):

$\mathrm{FTU}_{\mathrm{ns}}=\sum_{\mathrm{i}=1}^{\mathrm{g}} \mathrm{E}_{\mathrm{i}} * 5^{(\mathrm{i}-1) / 2}$

The FTU numbers range from 0 to 2020 .

The process of evaluating FTU from a subimage of $3 \times 3$ is shown in Fig.3.

\begin{tabular}{|l|l|l|}
\hline 90 & 130 & 145 \\
\hline 160 & 140 & 200 \\
\hline 100 & 140 & 250 \\
\hline
\end{tabular}$\rightarrow$\begin{tabular}{|l|l|l|}
\hline 0 & 1 & 3 \\
\hline 4 & & 4 \\
\hline 0 & 2 & 4 \\
\hline
\end{tabular}

(a)

(b)

$\mathrm{FTU}=\{0,1,3,4,4,2,0,4\}, \mathrm{FTU}_{\mathrm{n} 5}=1292$

(c)

Fig.3: (a) Original subimage (b) Representation of fuzzy texture elements (c) Evaluate FTU.

\section{PROPOSED METHOD FUZZY LEFT RIGHT TEXTURE UNIT MATRIX}

The texture spectrum method of texture analysis gives the texture information using eight neighbouring pixels around the central pixel. The level of this information depends on ordering of the neighbouring pixels. Further, a little work is reported $[18,20]$ in literature to produce strong texture information of an image by separating the neighboring pixels into groups and form a relationship between them. In the cross diagonal approach [21, 22], texture information of the image is evaluated by separating the neighbourhood pixels into diagonal and corner pixels. The corner pixels are not connected pixels. The cross diagonal approach is evaluated with original texture unit but not with the FTU information. To overcome that, LRTM is proposed [18]. It considers the minimum of the LTUM and RTUM. By this only one of the 8 combinations of LTUM and RTUM is considered. Therefore some of the useful information will be lost by not considering the remaining 7 combinations of LTUM and RTUM. To address this proposed method considers the average of LTUM and RTUM. This results an average fuzzy left and right texture unit (AFLRTU) by the proposed scheme.
In the proposed AFLRTU method of texture analysis, the eight elements in the texture unit are obtained from a neighborhood of $3 \times 3$ pixels. They are divided into two well connected equal groups containing four pixels named as Fuzzy Left Texture Unit (FLTU) and Fuzzy Right Texture Unit (FRTU) as shown in Fig.4. This FLRTU method further reduces the FTU from 2020 to 79. This reduction is useful for a good classification by reducing computational complexity.

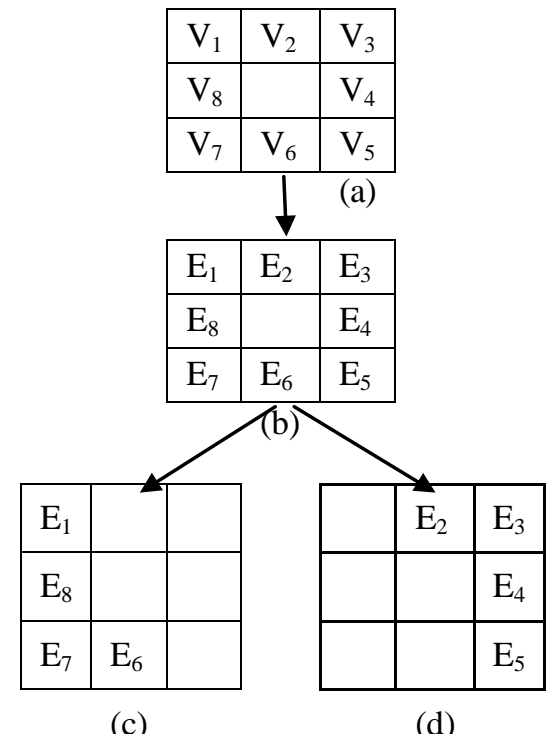

Fig.4: a) Representation of a) $3 \times 3$ neighbourhood b) Texture elements c) Formation of Fuzzy left texture unit (FLTU) matrix d) Formation of Fuzzy right texture unit (FRTU) matrix.

Each fuzzy texture element in the two groups has one of the five possible values $\{0,1,2,3$ and 4$\}$ as given in Equations (5) and (6):

$\mathrm{N}_{\text {FLIU }}=\sum_{\mathrm{i}=1}^{4} \mathrm{E}_{\mathrm{L}_{\mathrm{i}}} * 5^{(\mathrm{i}-1 / 2)}$
$\mathrm{N}_{\text {FRTU }}=\sum_{\mathrm{i}=1}^{4} \mathrm{E}_{\mathrm{R}_{\mathrm{i}}} * 5^{(\mathrm{i}-1 / 2)}$

where $\mathrm{N}_{\text {FLTU }}$ and $\mathrm{N}_{\text {FRTU }}$ are the fuzzy left texture unit number and fuzzy right texture unit number respectively. The $E_{\mathrm{Li}}$ and $\mathrm{E}_{\mathrm{Ri}}$ are the $\mathrm{i}^{\text {th }}$ element of left texture unit and right texture unit respectively. The entire process of transforming an image neighborhood into FLTU and FRTU is shown in Fig.5. The elements in the FLTU may be ordered in four different forms (i.e., $\left(E_{1}\right.$ to $\left.E_{6}\right)$ or $\left(E_{8}\right.$ to $\left.E_{1}\right)$ or $\left(E_{7}\right.$ to $\left.E_{8}\right)$ or $\left(E_{6}\right.$ to $\left.E_{7}\right)$ ) and FRTU may also be ordered in four different forms (i.e., $\left(E_{2}\right.$ to $\left.E_{5}\right)$ or $\left(E_{3}\right.$ to $\left.E_{2}\right)$ or $\left(E_{4}\right.$ to $\left.E_{3}\right)$ or $\left(E_{5}\right.$ to $\left.\left.E_{4}\right)\right)$ respectively. The values of FLTU and FRTU vary depending on the position of elements which can be labeled by using the Eqs. (5)\&(6). To overcome the problem of rotational invariance in FLTU and FRTU the minimum value of FLTU and FRTU is considered as the final FLTU and FRTU. Based on this the uniform FLTU and FRTU values of Fig.5 are the $\min (31,14,49,22)$ and $\min (72,43,52,66)$ respectively. 


\begin{tabular}{|l|l|l|}
\hline $0 \times 5^{0}$ & & \\
\hline $4 \times 5^{0.5}$ & & \\
\hline $0 \times 5^{1}$ & $2 \times 5^{1.5}$ & \\
\hline
\end{tabular}

\begin{tabular}{|l|l|l|}
\hline 0 & & \\
\hline 4 & & \\
\hline 0 & 2 & \\
\hline
\end{tabular}

\begin{tabular}{|c|c|c|}
\hline $0 \times 5^{1.5}$ & & \multirow{3}{*}{$\mathrm{N}_{\mathrm{FLTU}}=14$} \\
\hline $4 \times 5^{0}$ & & \\
\hline $0 \times 5^{0.5}$ & $2 \times 5^{1}$ & \\
\hline $0 \times 5^{1}$ & & \multirow{3}{*}{$\mathrm{N}_{\text {FLTU }}=49$} \\
\hline $4 \times 5^{1.5}$ & & \\
\hline $0 \times 5^{0}$ & $2 \times 5^{0.5}$ & \\
\hline
\end{tabular}

\begin{tabular}{|l|l|l|}
\hline $0 \times 5^{0.5}$ & & \\
\hline $4 \times 5^{1}$ & & \multirow{2}{*}{$N_{\text {FLTU }}=22$} \\
\hline $0 \times 5^{0.5}$ & $2 \times 5^{0}$ & \\
\hline \multicolumn{3}{|c|}{ Fig. 5 (a) }
\end{tabular}

Based taking the minimum value by one of the four combinations of FLTU and FRTU are considered. This process achieves rotational invariance but completely misses the other three combinations of FLTU and FRTU. The proposed AFLRTU method initially considers all the four possible FTU numbers from FLTU and FRTU. Then it finds the average value of FLTU (AFLTU) and average value of FRTU (AFRTU) numbers. By this the proposed AFLRTU achieves the rotational invariance and it considers the information from all fuzzy texture unit combinations. Both AFLTU and AFRTU range from 0:78. Then in the third stage, the proposed method finds the average of AFLTU and AFRTU. The proposed AFLRTU approach, maintains consistency with the AFLTU and AFRTU. In this way, a compromise is achieved between the conflicting requirements of greater statistical invariance on the one hand and greater discriminative power on the other.

\section{EXPERIMENTAL RESULTS}

In this experiment, three different datasets each of size $512 \times 512$ are used for analysis. Dataset- 1 contains 20 monochrome images obtained from VisTex colour image database and each texture image is subdivided into sixty four $64 \times 64$, sixteen $128 \times 128$ and four $256 \times 256$ nonoverlapping image regions. This leads to a total of 1680 (i.e., 20×84) sub-image regions that will be placed in the database. Dataset-2 is the full Brodatz texture database which contains 20 monochrome images while the Dataset-3 contains 20 monochrome images obtained from Granite colour image database. Since the number of images in the Dataset-3 is large, each texture image is subdivided into sixteen $128 \times 128$ and four $256 \times 256$ nonoverlapping image regions. It leads to a total of 400 (i.e., $20 \times 20$ ) sub-image regions of dataset-3.

The feature vector of first and second order statistical features are evaluated on the obtained FLTU and FRTU images. Feature vector $F_{1}$ contains the first order

\begin{tabular}{|l|l|l|}
\hline & $1 \times 5^{0}$ & $3 \times 5^{0.5}$ \\
\hline & & $4 \times 5^{1}$ \\
\hline & & $4 \times 5^{1.5}$ \\
$N_{\text {FRTU }}=72$
\end{tabular}

\begin{tabular}{|l|l|l|}
\hline & $1 \times 5^{1.5}$ & $3 \times 5^{0}$ \\
\hline & & $4 \times 5^{0.5}$ \\
\hline & & $4 \times 5^{1}$ \\
\hline & $1 \times 5^{1}$ & $3 \times 5^{1.5}$ \\
\hline & & $4 \times 5^{0}$ \\
\hline & & $4 \times 5^{0.5}$ \\
\hline
\end{tabular}$\quad N_{\text {FRTU }}=43$

\begin{tabular}{|l|l|l|}
\hline & $1 \times 5^{0.5}$ & $3 \times 5^{1}$ \\
\hline & & $4 \times 5^{1.5}$ \\
\hline & & $4 \times 5^{0}$ \\
\hline
\end{tabular} $\mathrm{N}_{\mathrm{FRTU}}=66$

Fig. 5 (b)

statistical features considered in the present approach are mean and standard deviation as given in Eqs.(7)\&(8). The feature vector $F_{2}$ contains second order statistical features such as energy, entropy, contrast, local homogeneity, correlation, cluster shade and cluster prominence as given in Equations (9)-(15).

mean $=\frac{1}{\mathbb{N}^{2}} \sum_{\mathrm{i} j=1}^{\mathbb{N}} \mathrm{P}\left(\mathbb{1}_{s} \mathbb{1}\right)$

Standard deviation $=\sqrt{\frac{1}{\mathbb{M}^{2}} \sum_{\mathrm{i} j=1}^{\mathrm{N}}\left[\mathrm{p}\left(\mathrm{i}_{s} \mathrm{j}\right)-\text { mean }\right]^{2}}$

Entropy $=\sum_{\mathrm{i} j=0}^{\mathbb{N}-1}-\ln \left(\mathrm{P}_{\mathrm{i} j}\right) \mathrm{P}_{\mathrm{Ij}}$

Energy $=\sum_{\mathrm{i} j=0}^{\mathbb{N}-1}-\ln \left(\mathrm{P}_{\mathrm{ij}}\right)^{2}$

Contrast $=\sum_{i=0}^{\mathbb{N}-1} P_{i j}(i-j)^{2}$

Local Homogenity $=\sum_{i, j=0}^{\mathbb{N}-1} \frac{P_{i j}}{1+(i-j)^{2}}$

Correlation $=\sum_{\mathrm{i} j=0}^{\mathbb{N}-1} \mathrm{P}_{\mathrm{ij}} \frac{(\mathrm{i}-\mu)(j-\mu)}{\sigma^{2}}$

where $P_{i j}$ is the pixel value in position $(i, j)$ of the texture image, $\mathrm{N}$ is the number of gray levels in the image, $\mu$ is $\mu=\sum_{i j=0}^{W-1} i P_{0}$ mean of the texture image and $\sigma^{2}=\sum_{i j=0}^{W-1} P_{i j}(i-\mu)^{2}$ variance of the texture image.

Cluster Shade $=\sum_{\mathrm{i} j=0}^{\mathrm{N}-1} \mathrm{P}_{\mathrm{ij}}\left(\mathrm{i}-\mathrm{M}_{\mathrm{N}}+\mathrm{j}-\mathrm{M}_{\mathrm{y}}\right)^{\mathrm{a}}$ 
Cluster Prominence $=\sum_{\mathrm{i} j=0}^{\mathrm{N}-1} \mathrm{P}_{\mathrm{i} j}\left(\mathrm{i}-\mathrm{M}_{\mathrm{x}}+\mathrm{j}-\mathrm{M}_{\mathrm{y}}\right)^{4}$

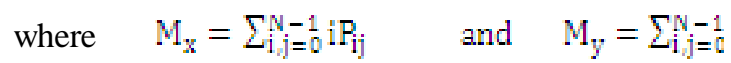

The above statistical features analyze the spatial distribution of gray values, by comparing local features at each point in the image. The reason behind this is the fact that the spatial distribution of gray values is one of the defining qualities of texture. In order to improve the classification gain the combination of feature vectors $F_{1}$ and $F_{2}$ is used as feature vector $F_{3}$ in the present paper.

In the classification phase, an unknown texture is used and its features are extracted and compared with the corresponding feature values stored in feature library using a Euclidean distance vector formula given in Eqn.(16).

Table 1.

\begin{tabular}{|c|c|c|c|c|}
\hline \multirow{2}{*}{ S.No } & \multirow{2}{*}{ Images } & \multicolumn{3}{|c|}{ Classification gain $(\%)$} \\
\hline & & $\mathrm{F}_{1}$ & $\mathrm{~F}_{2}$ & $\mathrm{~F}_{3}$ \\
\hline 1 & Bark.0006 & 92.9 & 96.4 & 95.2 \\
\hline 2 & Brick.0000 & 73.8 & 96.4 & 96.4 \\
\hline 3 & Brick.0004 & 77.4 & 81.0 & 78.6 \\
\hline 4 & Clouds.0001 & 91.7 & 96.4 & 94.1 \\
\hline 5 & Fabric. 0013 & 100.0 & 100.0 & 100.0 \\
\hline 6 & Fabric.0017 & 88.1 & 61.9 & 96.4 \\
\hline 7 & Flowers.0006 & 96.4 & 96.4 & 100.0 \\
\hline 8 & Food.0000 & 100.0 & 92.9 & 100.0 \\
\hline 9 & Food.0001 & 91.7 & 86.9 & 98.8 \\
\hline 10 & Grass.0001 & 100.0 & 61.9 & 92.9 \\
\hline 11 & Leaves.0012 & 96.4 & 95.2 & 100.0 \\
\hline 12 & Metal.0002 & 89.3 & 91.7 & 98.8 \\
\hline 13 & Metal.0004 & 97.6 & 97.6 & 100.0 \\
\hline 14 & Misc.0001 & 97.6 & 83.3 & 100.0 \\
\hline 15 & Misc. 0002 & 100.0 & 77.4 & 100.0 \\
\hline 16 & Sand.0000 & 95.2 & 57.1 & 96.4 \\
\hline 17 & Sand.0002 & 94.1 & 66.7 & 98.8 \\
\hline 18 & Tile. 0008 & 96.4 & 84.5 & 98.8 \\
\hline 19 & Water.0005 & 100.0 & 96.4 & 100.0 \\
\hline 20 & Wood.0002 & 100.0 & 90.5 & 100.0 \\
\hline \multicolumn{2}{|r|}{ Average } & 93.93 & 85.54 & 97.26 \\
\hline \multicolumn{2}{|c|}{$\begin{array}{l}\text { Number of image } \\
\text { regions correctly } \\
\text { classified }\end{array}$} & 1578 & 1437 & 1633 \\
\hline
\end{tabular}
second order features (1680 image regions)

\begin{tabular}{|c|c|c|c|c|}
\hline \multirow{2}{*}{ S.No } & \multirow{2}{*}{ Images } & \multicolumn{3}{|c|}{ Classification gain $(\%)$} \\
\hline & & $\mathrm{F}_{1}$ & $\mathrm{~F}_{2}$ & $\mathrm{~F}_{3}$ \\
\hline 1 & $\mathrm{D}_{1}$ & 95.83 & 91.67 & 100.0 \\
\hline 2 & $\mathrm{D}_{2}$ & 91.67 & 91.67 & 97.6 \\
\hline 3 & $\mathrm{D}_{3}$ & 95.83 & 91.67 & 78.6 \\
\hline 4 & $\mathrm{D}_{4}$ & 94.1 & 66.7 & 97.6 \\
\hline 5 & $\mathrm{D}_{5}$ & 96.4 & 84.5 & 100.0 \\
\hline 6 & $\mathrm{D}_{6}$ & 94.8 & 96.4 & 100.0 \\
\hline 7 & $\mathrm{D}_{7}$ & 90.7 & 93.4 & 100.0 \\
\hline 8 & $\mathrm{D}_{8}$ & 95.83 & 91.67 & 97.6 \\
\hline 9 & $\mathrm{D}_{9}$ & 91.7 & 86.9 & 97.6 \\
\hline 10 & $\mathrm{D}_{10}$ & 100.0 & 61.9 & 81.0 \\
\hline 11 & $\mathrm{D}_{11}$ & 96.4 & 95.2 & 100.0 \\
\hline 12 & $\mathrm{D}_{12}$ & 89.3 & 91.7 & 98.8 \\
\hline 13 & $\mathrm{D}_{13}$ & 97.6 & 97.6 & 100.0 \\
\hline 14 & $D_{15}$ & 97.6 & 83.3 & 98.8 \\
\hline 15 & $\mathrm{D}_{16}$ & 100.0 & 77.4 & 98.8 \\
\hline 16 & $D_{17}$ & 95.83 & 87.5 & 100.0 \\
\hline 17 & $\mathrm{D}_{18}$ & 87.5 & 87.5 & 97.8 \\
\hline 18 & $\mathrm{D}_{19}$ & 87.5 & 91.67 & 97.8 \\
\hline 19 & $\mathrm{D}_{20}$ & 91.67 & 91.67 & 96.5 \\
\hline 20 & D21 & 81.9 & 92.9 & 100.0 \\
\hline \multicolumn{2}{|c|}{ Average } & 93.61 & 87.65 & 96.93 \\
\hline \multicolumn{2}{|c|}{$\begin{array}{c}\text { Number of image } \\
\text { regions correctly } \\
\text { classified }\end{array}$} & 1564 & 1472 & 1628 \\
\hline
\end{tabular}

where $\mathrm{n}$ is the total number of features used, $\mathrm{i}=1$ to $\mathrm{Q}$

Using the feature vector $F_{1}, F 2, F_{3}$ the success rate achieved is $93.93 \%, 85.54,97.26 \%$ respectively. The classification result of Dataset-2 and Dataset-3 are given in the Table 2 and 3 respectively. The classification results exhibits the same tendency as Dataset-1

Table 2: Results of texture classification using Dataset-2 of first and 
Table 3: Results of texture classification using Dataset-3 of first and second order features (with 1680 image regions)

\begin{tabular}{|c|c|c|c|c|}
\hline \multirow{2}{*}{ S.No } & \multirow{2}{*}{ Images } & \multicolumn{3}{|c|}{ Classification gain $(\%)$} \\
\hline & & $\mathrm{F}_{1}$ & $\mathrm{~F}_{2}$ & $\mathrm{~F}_{3}$ \\
\hline 1 & $\mathrm{G}_{1}$ & 92.9 & 86.5 & 98.7 \\
\hline 2 & $\mathrm{G}_{2}$ & 94.1 & 94.7 & 97.6 \\
\hline 3 & $\mathrm{G}_{3}$ & 96.4 & 84.5 & 100.0 \\
\hline 4 & $\mathrm{G}_{4}$ & 89.5 & 92.9 & 97.6 \\
\hline 5 & $\mathrm{G}_{5}$ & 95.2 & 57.1 & 100.0 \\
\hline 6 & $\mathrm{G}_{6}$ & 94.1 & 66.7 & 97.6 \\
\hline 7 & $\mathrm{G}_{7}$ & 96.4 & 84.5 & 100.0 \\
\hline 8 & $\mathrm{G}_{8}$ & 100.0 & 92.9 & 97.6 \\
\hline 9 & $\mathrm{G}_{9}$ & 91.7 & 86.9 & 97.6 \\
\hline 10 & $\mathrm{G}_{10}$ & 95.8 & 91.7 & 100.0 \\
\hline 11 & $\mathrm{G}_{11}$ & 91.7 & 91.7 & 97.6 \\
\hline 12 & $\mathrm{G}_{12}$ & 95.8 & 91.7 & 78.6 \\
\hline 13 & $\mathrm{G}_{13}$ & 97.6 & 97.6 & 100.0 \\
\hline 14 & $\mathrm{G}_{14}$ & 98.5 & 83.3 & 98.8 \\
\hline 15 & $\mathrm{G}_{15}$ & 100.0 & 77.4 & 98.8 \\
\hline 16 & $\mathrm{G}_{16}$ & 95.2 & 57.1 & 100.0 \\
\hline 17 & $\mathrm{G}_{17}$ & 94.1 & 66.7 & 97.6 \\
\hline 18 & $\mathrm{G}_{18}$ & 96.4 & 84.5 & 100.0 \\
\hline 19 & $\mathrm{G}_{19}$ & 100.0 & 96.4 & 100.0 \\
\hline 20 & $\mathrm{G}_{20}$ & 100.0 & 90.5 & 100.0 \\
\hline \multicolumn{2}{|c|}{ Average } & 95.8 & 83.8 & 97.9 \\
\hline \multicolumn{2}{|c|}{$\begin{array}{c}\text { Number of image } \\
\text { regions correctly } \\
\text { classified }\end{array}$} & 1608 & 1407 & 1645 \\
\hline
\end{tabular}

\section{A. Comparision of the proposed FLRTUM with other Methods}

Table 1, 2 and 3 shows the mean percentage classification rate for each group of textures by using the proposed AFLRTU. Other existing methods of CDTS [21] and modified CDTS [22] method applied on VisTex, Brodatz and Granite texture database images are represented in Table 4 respectively. From Table 4, it is clearly evident that, the proposed AFLRTU exhibits a high classification rate than the existing methods. The graphical analysis of the percentage mean classification rate for the proposed AFLRTU and other existing methods of three databases are shown in Fig.6.
Table 4: Comparison of the proposed AFLRTU method with the existing methods.

\begin{tabular}{|l|c|c|c|}
\hline Database & CDTM & $\begin{array}{c}\text { Modified } \\
\text { CDTM }\end{array}$ & $\begin{array}{c}\text { Proposed } \\
\text { AFLRTU } \\
\text { Method }\end{array}$ \\
\hline VisTex & 84.92 & 92.6 & 96.79 \\
\hline Brodatz & 87.56 & 92.26 & 96.93 \\
\hline Granite & 75.71 & 90.32 & 97.90 \\
\hline
\end{tabular}

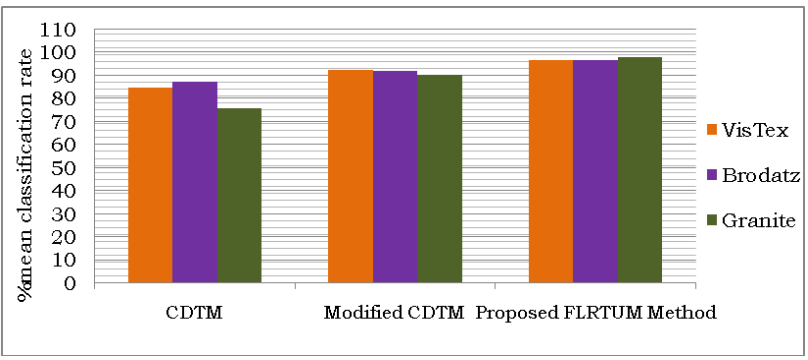

Fig.6: Comparison of proposed AFLRTU and existing methods.

\section{CONCLUSIONS}

The present paper derived a new AFLRTU to overcome the disadvantages of FTU, LRTM and CDTM. The AFLRTU approach considers two sets of four connected texture elements on a $3 \times 3$ grid for evaluating the TU instead of non-connected or corner texture elements as in the case of Cross Diagonal Texture Matrix (CDTM) or Modified CDTM. The AFLRTU reduced the size of TU from 2020 to 79 as in the case FTU, thus it reduced the overall complexity. The AFLRTU achieved rotational invariance by considering the average of all four combinations of TU, which is missing in LRTM. This feature extraction process is quite efficient with less complexity. When compared with other approaches, the proposed scheme is more effective and exhibiting increased classification ability by using smaller feature vectors. The experimental results prove the efficacy of the proposed method when compared to several other methods.

\section{ACKNOWLEDGMENT}

The authors would like to express their gratitude to Sri K.V.V. Satya Narayana Raju, Chairman, and K. Sashi Kiran Varma, Managing Director, Chaitanya group of Institutions for providing necessary infrastructure. Authors would like to thank anonymous reviewers for their valuable comments and Dr. G.V.S. Ananta Lakshmi for her invaluable suggestions which led to improvise the presentation quality of this paper 


\section{REFERENCES}

[1] Kaplan, L.M., Extended fractal analysis for texture classification and segmentation, IEEE Transactions on Image Processing, Vol. 8, No. 11, pp.1572-1585, 1999.

[2] R.M.Haralick, "Statistical and structural approaches to texture," Proc.IEEE, vol. 67, no. 5, pp. 786-804, 1979

[3] L.Kirvida, "Texture measurements for the automatic classification of imagery," IEEE Trans. Electromagnet. Compat, vol. 18, no. 2, pp. 38-42, 1976.

[4] A.Rosenfeld and M.Thurston, "Edge and curve detection for visual scene analysis," IEEE Trans. Comput, vol.20, no.5, pp. 562-569, 1971

[5] R.M.Haralick, K.Shanmugam, and I.Dinstein, "Texture features for image classi_cation," IEEE Trans.Syst.Man Cybernet, vol. 3, no. $\overline{11}$, pp. 610-621, 1973.

[6] S.Z.Li, "Markov random field modeling in computer vision," Springer-Verlag Tokyo, 1995

[7] A.Kundu and J.L.Chen, "Texture classification using gmf bank based sub band decomposition," CVGIP: Graphical models and image processing, vol. 54, no. 5, pp. 369-384, 1992.

[8] Wu, W.-R., Wei, S.-C.: Rotation and Gray-Scale Transform-Invariant Texture Classification Using Spiral Resampling, Subband Decomposition and Hidden Markov Model. IEEE Trans. Image Processing 5 (1996) 1423-1434.

[9] Chen, J.-L., Kundu, A.: Rotation and Gray Scale Transform Invariant Texture Identification Using Wavelet Decomposition and Hidden Markov Model. IEEE Trans. Pattern Analysis and Machine Intelligence 16 (1994) 208-214.

[10] D.C.He, L.Wang and J.Guibert, "Texture features extraction," Pattern Recognition Letters. Vol.6, pp.269- 273, 1987

[11] Dong-Chen He and Li Wang, “Texture unit, texture spectrum and Texture, IEEE Transactions on geoscience and Remote Sensing, Vol. 28, July 1990

[12] D.C.He and L.Wang, "Texture unit, texture spectrum and texture analysis," Proc of IGARSS.89, Vancouver, Canada, 1989, Vo1.5, pp.2769-2772

[13] L.Wang, D.C.He, "A new statistical approach to texture analysis," Photogrammetrics Eng. and Remote Sensing, pp.61-65, 1990

[14] L. Wang, D.C. He and A. Fabbri, "Textural filtering for SAR image processing," Proc. of IGARSS'89, Vancouver, Canada, 1989, Vol.5, pp. 2785-2788, 1989

[15] L.Wang and D.C.He, "Texture classification using texture spectrum," Pattern Recognition, 1989

[16] J.S. Taur, C.W. Tao, Texture classification using a fuzzy texture spectrum and neural networks, J. Electron. Imaging 7 (1) (1998) 29-35

[17] A.Barcelo, E.Montseny and P.Sobrevilla. “On Fuzzy Texture Spectrum for Natural Microtextures Characterization," Proceedings EUSFLAT-LFA, pp. 685-690, 2005
[18] Sujatha.B, Vijayakumar.V, Chandra Mohan M., "Rotationally Invariant Texture Classification using LRTM based on Fuzzy Approach," IJCA, vol.33, November 2011

[19] G. Wiselin Jiji a,, L. Ganesan, “A new approach for unsupervised segmentation," Applied Soft Computing 10 (2010) 689-693.

[20] M.Ramabai, V.Venkata Krishna, J.Sasi Kiran, "Morphological Shape features for Classification of Textures based on Fuzzy Texture Element," IJCA, Volume 19- No.7, April 2011

[21] Abdulrahman A. AL-JANOBI and AmarNishad M. THOTTAM, "Testing and Evaluation of CrossDiagonal Texture Matrix Method.

[22] Abdulrahman Al-Janobi, "Performance evaluation of cross-diagonal texture matrix method of texture analysis," Pattern Recognition, vol.34, pp: 171-180, 2001

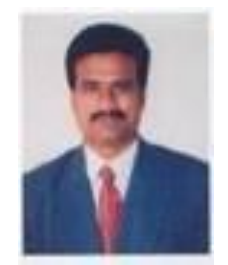

Y.Venkateswarlu obtained his MCA from VSM College, Andhra University in the year 1997 and M.Tech I.T (Information Technology) from Punjab University Patiala in the year $2003 \mathrm{He}$ is a member of SRRF-GIET, Rajahmundry. $\mathrm{He}$ is pursuing his Ph.D from AN University-Guntur in Computer Science \& Engineering under the guidance of Dr JVR Murthy He has published research papers in various National, Inter National conferences, proceedings. He has served the Chaitanya group of Engineering colleges for 12 years as Assistant Professor and Associate Professor and taught courses for MCA and M.Tech students. He has been HOD for Dept of CSE at Chaitanya Institute of Engineering and Technology, Rajahmundry since October 2012. He is a life member of ISCA

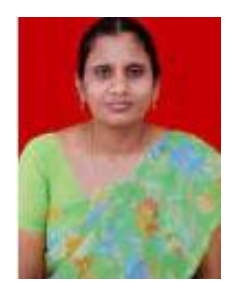

B.Sujatha received the B.Tech. degree from JNT University, Kakinada in 1997 and received her M. Tech. (Computer Science \& Engineering) from Andhra University in 2002. She is having 10 years of teaching experience. Presently she is working as an Assoc. Professor in GIET, Rajahmundary. She has published 20 research publications in Inter National Journal. She is a member of SRRF-GIET, Rajahmundry. She is pursuing her Ph.D from Mysore University in Computer Science under the guidance of Dr. V.Vijaya Kumar. Her research interest includes Image Processing and Pattern Recognition. She is a Life member of ISCA. 


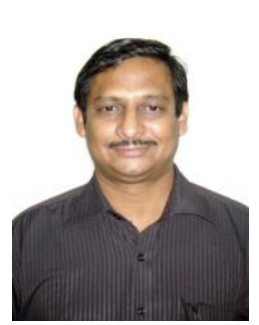

Dr.J.V.R.Murthy is a Professor and Chairman board of studies in the Department of Computer Science and Engineering, JNTU College of Engineering, Kakinada, A.P., INDIA. Dr. J.V.R.Murhy has specialized in Data warehousing and Mining. He has 24 years of Teaching, Research and more tha 3 years Industrial experience in USA as Senior People Soft Consultant at William M Mercer, Key Span Energy and AXA Client solutions.He is completed an R\&D project "The integration of META DATA Horizontal and Vertical traceability." In collaboration with BI divisions of TCS HYDERABAD and MUMBAI.He was awarded for "good performance and on schedule completion of People Soft HRMS project" by Key Span Energy Corporation, New York . He Played a very prominent role in a $\$ 20$ Million project for AXA Client solutions NewYork, $\mathrm{He}$ has a 25 research publications of international Journals/Conferences including IEEE \& Elsevier sciences 